\title{
Evaluación de Tres Formulaciones Comerciales de Aplicación Pour on Bajo Condiciones de Campo y su Efecto in vitro en el Control de Boophilus microplus (Acari: Ixodidae) en Bovinos de Ceja de Selva
}

\author{
Evaluation of Three Pour on Commercial Formulations under Field Conditions \\ AND THEIR IN VITRo EFFECT ON THE CONTROL OF Boophilus microplus (ACARI: \\ IXodidae) in CaTtLe in Tropical LoWLANDS
}

César del Castillo L. ${ }^{1,3}$, Rosa Pinedo V. ${ }^{1}$, Luis Rodríguez I. ${ }^{2}$, Amanda Chávez V. ${ }^{1,4}$

\section{Resumen}

\begin{abstract}
El objetivo del presente estudio fue evaluar la eficacia de tres formulaciones comerciales pour on bajo condiciones de campo e in vitro sobre el control de Rhipicephalus (Boophilus) microplus en bovinos naturalmente infestados, en la zona de Oxapampa, Perú. Se trabajó con 105 bovinos infestados con 30 o más teleoginas en un flanco. Los animales se distribuyeron en tres grupos de 35 animales c/u tratados con Fipronil 1\% + Abamectina $0.5 \%$ + Aceite de Neem 2\% (FAN), Flumetrina 1\% + Eprinomectina $0.5 \%$ (FE) y Flumetrina $1 \%$ + Fluazurón $2.5 \%(\mathrm{FF})$, respectivamente. La dosis empleada fue de $1 \mathrm{ml} / 10 \mathrm{~kg}$ p.v en la línea dorsal (pour on). Se contó el número de teleoginas $(\geq 4.5 \mathrm{~mm}$ ) sobre los animales los días $0,7,14,21,28$ y 56 postratamiento. Se calculó la efícacia mediante la prueba de reducción de recuento de teleoginas. Para el estudio in vitro se colectaron 50 teleoginas de cada grupo a las 24,48 y 72 h postratamiento, así como de un grupo no dosificado. Se inmovilizaron con cinta adhesiva 30 teleoginas de pesos similares (160-340 mg) por grupo y se incubaron a $27^{\circ} \mathrm{C}$ y $85 \%$ de humedad relativa por 21 días. Se evaluó mortalidad y oviposición. Los huevos se incubaron por 30 días para determinar la eclosión larval, eficiencia reproductiva y eficacia in vitro. El estudio en campo demostró que las combinaciones FAN (99.4-100\%), FE (95.8-98.4\%) y FF (97.3-98.8\%) fueron eficaces (>95\%) los días 7-28, 7-14 y 21-56, respectivamente. In vitro, las combinaciones FAN y FF tuvieron $100 \%$ de eficacia en las tres colectas; mientras que la combinación FE mostró una eficacia del $98-99 \%$ en teleoginas colectadas a las 48 y 72 h postratamiento.
\end{abstract}

Palabras clave: fipronil, flumetrina, eprinomectina, fluazurón, teleoginas, Oxapampa

\footnotetext{
${ }^{1}$ Laboratorio de Microbiología y Parasitología Veterinaria, Facultad de Medicina Veterinaria, Universidad Nacional Mayor de San Marcos, Lima, Perú

${ }^{2}$ Unidad de Negocio Sanivet-Montana, Lima, Perú

${ }^{3}$ E-mail: cdelcastilloll@gmail.com

${ }^{4}$ E-mail: achavezvg@gmail.com
} 
The aim of this study was to evaluate three pour on commercial formulations under field and in vitro conditions on the control of Rhipicephalus (Boophilus) microplus in naturally infested cattle in Oxapampa, Peru. For the field evaluation 105 cattle having 30 or more engorged female ticks in one flank were selected and equally distributed in three groups of 35 animal each: Fipronil 1\% + Abamectin $0.5 \%+$ Neem Oil 2\% (FAN), Flumethrin $1 \%+$ Eprinomectin $0.5 \%$ (FE), and Flumethrin 1\% + Fluazuron $2.5 \%$ (FF). All animals were treated with $1 \mathrm{ml} / 10 \mathrm{~kg}$ bw via pour on the dorsal line. The number of $\geq 4.5 \mathrm{~mm}$ engorged female was counted on days $0,7,14,21,28$, and 56 post-treatment. Effectiveness was evaluated by the Teleogines Count Reduction Test. For the in vitro study 50 teleogines (160-340 mg) were collected per group at 24, 48, and $72 \mathrm{~h}$ post-treatment, as well as from a separate group without treatment. Thirty ticks of similar weights (160-340 mg) per group were immobilized with adhesive tape and incubated at $27^{\circ} \mathrm{C}$ and $85 \%$ relative humidity during 21 days. Mortality and oviposition were evaluated. The eggs were incubated during 30 days to determine larval hatching, reproductive efficiency and in vitro effectiveness. The field study showed that FAN (99.4-100\%), FE (95.8-98.4\%), and FF (97.3-98.8\%) had adequate efficacy (>95\%) on days 7 to 28,7 to 14 and 21 to 56 , respectively. In the in vitro evaluation only FAN and FF showed $100 \%$ efficacy on engorged female collected at 24,48 , and $72 \mathrm{~h}$ post-treatment, while FE showed an efficacy of $98-99 \%$ on engorged female collected at 48 and $72 \mathrm{~h}$ post-treatment.

Key words: fipronil, flumethrin, eprinomectin, fluazuron, teleogines, Oxapampa

\section{INTRODUCCIÓN}

La garrapata Rhipicephalus (Boophilus) microplus (Acari: Ixodidae) denominada «garrapata común del ganado», es el ectoparásito más importante en áreas de explotación pecuaria de regiones tropicales y subtropicales (Manual Merck, 2000), debido a su impacto en la salud bovina, especialmente por su rol como vector de hemoparásitos (Barriga, 2002). Además, es responsable de severas pérdidas económicas por mermas en la producción de leche, ganancia de peso y por daños a las pieles y cueros por la acción traumática de las picaduras (FAO, 1984; Urquhart et al., 2001; Rojas, 2004; Casas et al., 2009). En el Perú se encuentra distribuida en las zonas tropicales y subtropicales de la costa norte, valles interandinos, y selva alta y baja.
En su ciclo de vida interviene un solo hospedero; es decir, desarrolla todos sus estadios de vida sobre el mismo animal, siendo este periodo de 17 a 25 días en ganado Bos taurus. La fase de vida libre o no parasítica se inicia cuando la hembra adulta ingurgitada o teleogina se desprende del animal para realizar la oviposición en el medio ambiente, donde llega a producir entre 1500 a 5000 huevos. El periodo de postura varía entre 2 a 36 días, pudiendo llegar hasta 40 días en temperaturas inferiores de $15{ }^{\circ} \mathrm{C}$ (Núñez et al., 1982; Cordero del Campillo et al., 1999).

Para su control se han venido empleando diversos ixodicidas, como los arsenicales, organoclorados, organofosforados, carbamatos, formamidas, piretroides sintéticos (flumetrina), lactonas macrocíclicas (eprinomectina), y fenilpirazoles (fipronil); así como algunos reguladores del crecimiento 
como los análogos de la hormona juvenil (metopreno y fenoxicarb) y los inhibidores de la síntesis de quitina, como el fluazurón, diflubenzurón, lufenurón y la ciromazina (Cordero del Campillo et al., 1999; Cuore et al., 2008; Rodríguez-Vivas et al., 2010). Los ixodicidas han sido utilizados con éxito en el control de las garrapatas; sin embargo, su uso continuo e irracional ha generado cepas resistentes. Desde años atrás se ha reportado la presencia de resistencia a los arsénicos, clorinados, carbamatos, organofosforados, piretroides sintéticos, amitraz y, más recientemente, a la ivermectina y fipronil (Santamaría et al., 2003; Perez-Cogollo et al., 2010).

Las vías de aplicación de los garrapaticidas son múltiples (baños de inmersión, aspersión e inyectable); algunas de las cuales requieren de infraestructura y mayor uso de personal. En contraste, la aplicación «pour on» o epicutánea consiste en derramar el producto sobre la línea medial dorsal del bovino, desde la cruz hasta la base de la cola, por lo que es de fácil aplicación, de un manejo más simple de los animales y requiere menor número de personal para su aplicación (Stendel et al., 1992).

Dentro de las drogas de reciente introducción al mercado se menciona a flumetrina y fluazurón, drogas de mayor eficacia (Mekonnen, 2000; Tang et al., 2008). Asimismo, fluazurón ofrece una mayor residualidad. Debido a esto, se hace necesario evaluar nuevas formulaciones comerciales de fácil aplicación en el control de $R$. microplus. Es así que el objetivo del presente estudio fue evaluar la eficacia de tres formulaciones comerciales pour on bajo condiciones de campo e in vitro sobre el control de $R$. microplus en bovinos naturalmente infestados, de la zona de ceja de selva, Oxapampa, Perú.

\section{Materiales y Métodos}

\section{Lugar y Animales}

El estudio se realizó en el distrito de Oxapampa, ubicado en el lado amazónico de los Andes centrales peruanos y en la porción oriental de la región Pasco. Presenta un clima húmedo, semicálido y con temperaturas promedio de 13 a $20^{\circ} \mathrm{C}$ (Municipalidad Provincial de Oxapampa, 2010).

Se trabajó con ocho fundos ganaderos, con animales y manejos similares. Se seleccionaron 105 bovinos adultos, machos y hembras, para la evaluación en campo y 40 para la evaluación in vitro. Todos los animales eran cruces de Holstein y Brown Swiss, que se encontraban infestados con garrapatas. Como criterio de inclusión, los animales debían presentar un mínimo de 30 garrapatas de 4.5 a $8 \mathrm{~mm}$ sobre un lado del cuerpo (representado por el $50 \%$ del cuerpo e incluye la cabeza, cuello, tórax, abdomen y miembros, sea del flanco derecho o izquierdo) y como criterios de exclusión, los animales que recibieron tratamiento de garrapaticidas en los últimos tres meses. Todos los animales permanecieron en sus respectivos fundos recibiendo el mismo manejo y alimentación por parte de sus propietarios. El estudio se realizó durante los meses de febrero, mayo y junio de 2012.

\section{Tratamientos}

Se utilizaron tres formulaciones comerciales, compuestos por combinación de principios activos: Fipronil 1\% + Abamectina $0.5 \%$ + Aceite de Neem 2\% (FAN), Flumetrina $1 \%$ + Eprinomectina $0.5 \%$ (FE), y Flumetrina $1 \%$ + Fluazurón $2.5 \%$ (FF), teniéndose 35 animales por tratamiento. La dosificación se hizo de acuerdo a las dosis recomendadas por el laboratorio fabricante. 
Se vertió $1 \mathrm{ml} / 10 \mathrm{~kg}$ p.v (pour on) sobre la línea media dorsal, por delante de la escápula hasta la base de la cola. El peso vivo fue determinado mediante una cinta bovinométrica. Se registró la identificación del animal, grupo experimental, y número de teleoginas presentes antes y después del tratamiento.

\section{Evaluación en Campo}

\section{Recuento de teleoginas}

La distribución de los animales entre los tres tratamientos se hizo en forma equitativa y proporcional al recuento de garrapatas. El conteo de teleoginas presentes en un lado del cuerpo de los animales se hizo en el día 0 (día del tratamiento) y en los días 7, 14, 21, 28 y 56 postratamiento. El día 0 fue considerado como grupo control.

\section{Porcentaje de eficacia}

La eficacia de los garrapaticidas se calculó mediante la prueba de reducción de recuento de garrapatas, la cual considera una relación porcentual entre la reducción del promedio de garrapatas en los grupos tratados con respecto al promedio del día 0 , mediante la siguiente fórmula (Wood et al., 1995):

$\operatorname{Eficacia}(\%)=\frac{\left(X_{d=0}\right)-\left(X_{d=7,14,21,28,56}\right)}{\left(X_{d=0}\right)} \times 100$

La eficacia de un producto comercial en campo al día 21 debe ser e»95\%, y en los casos donde la acción acaricida es de lento inicio, el porcentaje de eficacia debe ser calculado por encima de los 21 días (Holdsworth et al., 2006).

\section{Evaluación in vitro}

\section{Manejo de las teleoginas}

Se colectaron manualmente 50 teleoginas de 10 bovinos no tratados, y que conformaron el grupo control. Asimismo, se colectaron 50 teleoginas de cada uno de los otros grupos experimentales a las 24, 48 y 72 horas postratamiento. Los ácaros fueron transportados al Laboratorio de Parasitología de la Facultad de Medicina Veterinaria, Universidad Nacional Mayor de San Marcos, Lima.

De las 50 teleoginas de cada grupo experimental se seleccionaron 30 de ellas con pesos entre 160 y $340 \mathrm{mg}$ (Drummond et al., 1973), utilizando una balanza analítica con precisión de $0.0001 \mathrm{~g}$. Se formaron tres grupos de 10 teleoginas c/u con pesos similares, siendo inmovilizadas en placas petri con tiras de cinta adhesivas adheridas por la parte caudodorsal del cuerpo. Las placas con teleoginas fueron identificadas (fecha, tratamiento y peso de las teleoginas) y acondicionadas en un ambiente climatizado a $27^{\circ} \mathrm{C}$ y $85 \%$ de humedad relativa.

\section{Parámetros evaluados}

- Porcentaje de mortalidad y oviposición: Las teleoginas fueron observadas diariamente a través de un estereoscopio, viendo la presencia de signos vitales como: movimientos de patas y de asas intestinales (Rodríguez et al., 2010), a fin de determinar su vitalidad y oviposición postratamiento hasta el día 21 de su colecta, Además, se pesó el total de huevos de cada grupo experimental.

- Porcentaje de eclosión: Los huevos fueron colectados y acondicionados en tubos de Metianiu (Nuñez et al., 1982), y mantenidos en incubación $\left(27^{\circ} \mathrm{C}\right.$ y $85 \%$ de humedad relativa) por 30 días. Se estimó la eclosión larval mediante observación directa de los huevos no eclosionados y de las larvas presentes.

- Determinación de la eficiencia reproductiva (ER) y eficacia de los tratamientos $(E)$ : La eficiencia reproductiva (ER) expresa la habilidad de una teleogina para transformar parte de su peso inicial en larvas viables. Se calcula dividiendo el peso de la masa total de huevos entre el peso inicial de la teleogina 
y el valor obtenido se multiplica por el porcentaje de eclosión. La eficacia de los tratamientos (E) expresa la diferencia porcentual entre las eficiencias reproductivas del grupo tratado y control. Para esto, se usaron las siguientes fórmulas (Drummond et al., 1973):

$$
\begin{aligned}
\mathrm{ER} & =\frac{\text { Peso de los huevos }(\mathrm{g}) \times \text { Eclosión }}{\text { Peso de las teleoginas }(\mathrm{g})} \\
\mathrm{E} & =\frac{(\text { ER control }- \text { ER tratado })}{\text { ER control }} \times 100
\end{aligned}
$$

\section{Análisis Estadístico}

Se utilizó la prueba de Kruskal-Wallis para determinar si hubo identidad o diferencia en las medianas de los tratamientos.

\section{Resultados}

En el Cuadro 1 se muestra la eficacia en campo de los ixodicidas comerciales evaluados, donde el tratamiento FAN resultó efi- caz $(\geq 95 \%)$ del día 7 al 28 postratamiento (PT), la combinación FE solo resultó ser eficaz los días 7 y 14 PT, en tanto que el grupo FF alcanza una eficacia de $94.4 \%$ el día 14 , y del día 21 al 56 PT muestra una eficacia satisfactoria en el control de las garrapatas.

El Cuadro 2 muestra la eficacia garrapaticida in vitro, evaluadas a través de mortalidad, oviposición y peso de los huevos a los 21 días poscolecta, y a la eclosión a los 30 días poscolecta. Los porcentajes de mortalidad de las teleoginas de $R$. microplus con el tratamiento de FAN fueron superiores al $80 \%$ a partir de la 24 horas PT, incrementándose al $100 \%$ a las 72 horas PT. Por otro lado, las mortalidades en el caso de FE y FF oscilaron entre el 13.3 a $33.3 \%$.

Con respecto a oviposición y eclosión de huevos de las teleoginas, la combinación FAN presentó $0 \%$ de oviposición desde las 24 horas PT, mientras que los porcentajes de oviposición variaron de 43 a $70 \%$ para FE y de 60 a $73.3 \%$ para FF, durante los tiempos evaluados. Con respecto a la eclosión de los huevos, solo las provenientes de las teleoginas tratadas con FE presentaron porcentajes iguales o superiores al $50 \%$.

Cuadro 1. Eficacia del tratamiento con Fipronil + Abamectina + Aceite de Neem (FAN; $\mathrm{n}=35)$, Flumetrina + Eprinomectina $(\mathrm{FE} ; \mathrm{n}=25)$ y Fluazurón + Flumetrina (FF; $\mathrm{n}=21$ ) en bovinos infestados naturalmente con garrapatas Rhipicephalus (Boophilus) microplus en Oxapampa, Perú (2012)

\begin{tabular}{ccccccc}
\hline \multirow{2}{*}{$\begin{array}{c}\text { Días pos- } \\
\text { tratamiento }\end{array}$} & \multicolumn{2}{c}{ FAN } & \multicolumn{2}{c}{ FE } & \multicolumn{2}{c}{ FF } \\
& $\begin{array}{c}\text { Promedio }^{1} \\
\text { de garrapatas }\end{array}$ & $\begin{array}{c}\text { Eficacia } \\
(\%)\end{array}$ & $\begin{array}{c}\text { Promedio }^{1} \\
\text { de garrapatas }^{1}\end{array}$ & $\begin{array}{c}\text { Eficacia } \\
(\%)\end{array}$ & $\begin{array}{c}\text { Promedio }^{1} \\
\text { de garrapatas }^{\text {Eficacia }} \\
(\%)\end{array}$ \\
\hline 0 & 38.03 & --- & 36.68 & --- & 31.38 & --- \\
7 & 0.03 & $99.9^{\mathrm{a}}$ & 0.60 & $98.4^{\mathrm{a}}$ & 8.75 & $72.1^{\mathrm{b}}$ \\
14 & 0.00 & $100.0^{\mathrm{a}}$ & 1.55 & $95.8^{\mathrm{b}}$ & 1.77 & $94.4^{\mathrm{b}}$ \\
21 & 0.00 & $100.0^{\mathrm{a}}$ & 2.05 & $94.4^{\mathrm{b}}$ & 0.37 & $98.8^{\mathrm{a}}$ \\
28 & 0.21 & $99.4^{\mathrm{a}}$ & 2.13 & $94.2^{\mathrm{b}}$ & 0.50 & $98.4^{\mathrm{a}}$ \\
56 & 4.43 & $88.3^{\mathrm{a}}$ & 2.80 & $92.4^{\mathrm{b}}$ & 0.85 & $97.3^{\mathrm{c}}$ \\
\hline
\end{tabular}

${ }^{1}$ Promedio aritmético

a,b,c Superíndices diferentes dentro de filas indican diferencia estadística $(p \varangle 0.05)$ 
Cuadro 2. Eficacia garrapaticida in vitro sobre 30 teleoginas de Rhipicephalus (Boophilus) microplus colectadas a las 24,48 y 72 horas del tratamiento provenientes de bovinos tratados con tres formulaciones comerciales pour on

\begin{tabular}{|c|c|c|c|c|c|c|}
\hline $\begin{array}{c}\text { Grupo/ } \\
\text { horas }\end{array}$ & $\begin{array}{l}\text { Mortalidad } \\
(\%)^{1}\end{array}$ & $\begin{array}{l}\text { Oviposición } \\
(\%)^{1}\end{array}$ & $\begin{array}{c}\text { Peso de } \\
\text { huevos } \\
(\mathrm{g})^{1}\end{array}$ & $\begin{array}{l}\text { Eclosión } \\
(\%)^{2}\end{array}$ & $\begin{array}{l}\text { Eficiencia } \\
\text { reproductiva }\end{array}$ & $\begin{array}{c}\text { Eficacia } \\
(\%)\end{array}$ \\
\hline Control & 0 & 100 & 2.64 & 95.25 & 33.46 & 0 \\
\hline \multicolumn{7}{|l|}{ FAN $^{3}$} \\
\hline 24 & 83.3 & 0 & 0 & 0 & 0 & 100 \\
\hline 48 & 96.7 & 0 & 0 & 0 & 0 & 100 \\
\hline 72 & 100 & 0 & 0 & 0 & 0 & 100 \\
\hline \multicolumn{7}{|l|}{$\mathrm{FE}^{3}$} \\
\hline 24 & 33.3 & 70 & 0.20 & 70 & 4.34 & 87 \\
\hline 48 & 30 & 43 & 0.07 & 65 & 0.67 & 98 \\
\hline 72 & 33.3 & 43 & 0.02 & 50 & 0.19 & 99 \\
\hline \multicolumn{7}{|l|}{$\mathrm{FF}^{3}$} \\
\hline 24 & 16.7 & 60 & 0.64 & 0 & 0 & 100 \\
\hline 48 & 13.3 & 73.3 & 0.67 & 0 & 0 & 100 \\
\hline 72 & 16.7 & 60 & 0.62 & 0 & 0 & 100 \\
\hline
\end{tabular}

La eficiencia reproductiva de las teleoginas para los grupos FAN y FF fue de cero. Asimismo, el porcentaje de eficacia los grupos FAN y FF mostraron ser eficaces en un $100 \%$ a partir de las 24 horas PT, en tanto que el grupo FE alcanzó una eficacia del 98 y $99 \%$ en las teleoginas colectadas a las 48 y 72 horas PT.

\section{Discusión}

Los resultados muestran que la combinación FAN (Fipronil 1\% + Abamectina 0.5\% + Aceite de Neem 2\%) fue eficaz (99.4 a $100 \%$ ) en el control de campo desde el día 7 al día 28 PT. Es posible que la combinación de estos principios activos haya originado la alta eficacia; sin embargo, evaluaciones individuales de sus componentes pueden explicar los resultados obtenidos. Así, se conoce que el fipronil, desde su introducción al mercado veterinario, ha demostrado niveles satisfactorios de eficacia y largos periodos residuales en caninos y bovinos, siendo hasta dos meses en el bovino (Davey et al., 1998; Da Silva, 2008; Penrroz, 2009), debido a su liberación gradual vía ductos foliculares, ya que se fija y almacena en las glándulas sebáceas dado su carácter altamente lipofílico (Muñoz, 2002). Lopes et al. (2014), asimismo, obtuvieron una eficacia de $99 \%$ desde el día 7 al 28 PT, resultados semejantes al presente estudio. 
La abamectina, lactona macrocíclica, posee un efecto tóxico sobre los ecto- y endoparásitos, siendo más conocida su eficacia contra nematodos y ligeramente menos activo frente a $R$. microplus (Shoop et al., 1995). Se absorbe y distribuye en tejidos como grasa, piel y mucosa intestinal debido a su lipofilia (Díaz, 2012). Así, Da Silva (2008), utilizando abamectina al $0.5 \%$ encontró una eficacia de 92 y $84 \%$ hacia el día 13 y 27 PT, respectivamente, en tanto que Pereira (2009) indica una eficacia de $80 \%$ hacia el día 21 PT con abamectina al $1 \%$.

El aceite de Neem (nim) es un repelente, insecticida y acaricida, con formulaciones comerciales en los países asiáticos de donde es nativa la planta (Williams y Mansingh, 1996). Sus compuestos aislados manifiestan sus efectos en el organismo como disuasivo durante la alimentación, perjudicando además la utilización de los alimentos ingeridos al actuar como inhibidor de la actividad enzimática del tubo digestivo, afectando la conversión alimentaria (Martínez y Van Endem, 1999; Martínez, 2002). Bezerra et al. (2009) utilizaron un pulverizado de este aceite al $1 \%$ y obtuvieron una eficacia del 92 y $84 \%$ al día 7 y 14 PT, respectivamente, frente a infestaciones naturales con $R$. microplus.

Las eficacias mostradas con el solo uso de abamectina o de nim son inferiores en comparación a los resultados obtenidos con la asociación FAN; de allí que es posible que el principal factor para lograr la eficacia alcanzada sea debida al fipronil; en tanto que la abamectina reforzaría el control sobre las garrapatas y sobre los parásitos internos por su acción endectocida. Asimismo, el nim colaboraría en el control inicial de ectoparásitos debido a su acción de repelencia. Por lo tanto, FAN podría emplearse en animales jóvenes que presentan infestaciones por garrapatas y endoparásitos, así como también sobre animales en engorde o en periodo de seca.

La asociación FE (Flumetrina 1\%+ Eprinomectina 0.5\%) en el ensayo de campo, mostró una adecuada eficacia en los pri- meros 14 días PT, decayendo hasta $92.4 \%$ el día 56 PT. Mekonnen (2000) al evaluar flumetrina en campo halló un 100\% de eficacia desde el día 4 al 33 PT, resultando ineficaz al día 40. La flumetrina administrada vía percutánea se absorbe escasamente a través de la piel, por lo que mayormente permanece al exterior del tejido adiposo, actuando por contacto directo con los artrópodos (Elliott et al., 1978).

Por su parte, Aguirre et al. (2005) obtuvieron resultados donde la eprinomectina al $0.5 \%$ inicia con una eficacia de $86.9 \%$ al día 7 y termina con una eficacia de $96.1 \%$ al día 28 PT, difiriendo con los resultados obtenidos en el presente estudio. La acción endectocida de la eprinomectina se debe al elevado porcentaje de unión a proteínas plasmáticas, concentrándose principalmente en grasa e hígado, lo cual favorece su control sobre endo- y ectoparásitos los primeros siete días del tratamiento (Plumb, 2010). La FE representa una buena alternativa al presentar una eficacia mayor del $95 \%$ y mantener un mayor periodo de eficacia por encima del $90 \%$. Además, se puede emplear en vacas en lactación ya que sus principios activos no requieren tiempo de retiro en leche debido a su farmacocinética (Baoliang et al., 2006; Villar et al., 2012).

La combinación FF (Flumetrina 1\% + Fluazurón $2.5 \%$ ) mostró una eficacia inferior al $95 \%$ al día 7 PT. Sin embargo, se incrementó a partir de los 14 días PT, evidenciando una eficacia satisfactoria desde el día $21(98.8 \%)$ al $56(97.3 \%)$. Además, el efecto residual óptimo (97.3\%) lo presentó el día 56 PT. Esta combinación, a pesar de tener flumetrina en su composición, no evidenció el $100 \%$ de eficacia obtenida con este principio por Mekonnen (2000) en el día 4 PT.

El efecto residual observado a las ocho semanas del tratamiento se debe básicamente al efecto del fluazurón, que se caracteriza por interferir principalmente en la síntesis y depósito de quitina, impidiendo así la formación de cutícula en el ectoparásito. Esto con- 
lleva a la incapacidad de promover la ecdisis (Muñoz, 2002), interrumpiéndose el desarrollo de los estadios evolutivos y disminuyendo la cantidad de adultos a partir de la tercera semana del tratamiento.

El fluazurón es absorbido mediante el lamido y, de manera lenta, a través de la piel, originando su almacenamiento en el tejido adiposo y una liberación lenta hacia el torrente sanguíneo. Esto permite mantener niveles eficaces en sangre durante más de 11 semanas (EMEA, 2005; Casas et al., 2009). La persistencia del fármaco en el bovino no es recomendable para vacas de alta producción, siendo su uso principalmente en vacas de seca, ganado de engorde y terneros.

En el estudio in vitro se pudo observar que la combinación FAN mostro una eficacia del $100 \%$ en su acción garrapaticida sobre teleoginas y esto va asociado con el $100 \%$ de mortalidad a las 72 horas PT y a una nula oviposición.

En el caso de FAN, se conoce que el fipronil actúa inhibiendo el impulso nervioso en los invertebrados, originando así hiperexcitabilidad, parálisis y muerte (CastroJaner et al., 2010; Prullage et al., 2011). Evaluaciones in vitro fueron realizadas por Davey et al. (1998) con fipronil pour on al 1\%, donde obtuvieron una eficacia de $99.7 \%$ durante 8 semanas PT; siendo estos resultados cercanos al 98.3\% hallado por Cid et al. (2012) mediante la prueba de inmersión (400ppm). Dichos resultados son similares a los obtenidos en el presente estudio, a pesar de no haber usado la misma metodología por tratarse de productos aplicados en forma pour on. La ausencia de oviposición puede explicarse por el estudio de Oliveira et al. (2009), quienes mencionan que los ovocitos sufren cambios morfológicos que determinan su muerte celular.

En un estudio, la abamectina in vitro produjo una reducción en la oviposición de $37 \%$ y una eclosión de 33\% (Pereira, 2009), en tanto que Cid et al. (2010) obtuvo 90.4\% de eficacia. Estos resultados son indicativos de un control parcial del ciclo biológico, pero es evidente que la mayor acción recae sobre el mecanismo del fipronil.

Por otra parte, la influencia del aceite de nim sería menor, tal y como se demostró en otros trabajos donde se obtuvieron mortalidades 65 a 68\% (Mansingh y Williams, 1998; Broglio-Micheletti et al., 2009). En forma similar, Costa et al. (2008) obtuvieron una eficacia in vitro de $32 \%$, que está por debajo del valor mínimo de $95 \%$ establecido por Holdsworth et al. (2006).

La asociación $\mathrm{FE}$ en el ensayo in vitro mostró una eficacia del $99 \%$ a las 72 horas PT, a pesar de obtener parámetros insatisfactorios en mortalidad de teleoginas (33.3\%), oviposición (43\%) y eclosión de huevos (65\%). Lo más resaltante fue la considerable reducción de la masa de huevos $(0.02 \mathrm{~g})$ a las 72 horas PT, razón por la cual mejoró notablemente el porcentaje de eficacia. Por otro lado, la eficacia podría explicarse por la acción de contacto de la flumetrina y la actividad sistémica de la eprinomectina. Resultados similares fueron hallados por Elias (1987), El-Azazy y Lucas (1996) y Cuore $e t$ al. (2009).

A pesar que la flumetrina actúa por contacto y permanece sobre el hospedero, su efecto «knock down» fue pobre. La literatura describe que puede ocasionar una hiperexitabilidad, por lo cual algunas garrapatas podrían desprenderse del animal y posteriormente retomar sus funciones vitales (Muñoz, 2002). El efecto letal débil se debe a que fluazurón solo tiene un efecto garrapaticida de $3.2 \%$ sobre ninfas (Oliveira et al., 2012).

La ausencia de eclosión se debe a la acción del fluazurón, que inhibe la síntesis de quitina, lo que reprime la viabilidad larval durante la eclosión (EMEA, 2005; Casas et al., 2009; Oliveira et al., 2012). Por otra parte, la reducción de la oviposición y de la masa de huevos es producido únicamente por la ac- 
ción inhibitoria de la flumetrina (El-Azazy y Lucas, 1996).

Por lo resultados mostrados tanto en campo como in vitro se puede considerar que la asociación FAN controla la presencia de teleoginas hasta el día $28 \mathrm{PT}$, siendo su uso recomendable sobre animales en periodo de seca, animales jóvenes o de carne en los que se respete el tiempo de retiro. La asociación FE controla la presencia de teleoginas en campo hasta el día 14 PT, teniendo como ventaja adicional, la ausencia de tiempo de retiro en leche de modo que puede ser empleada en animales en lactación. La asociación FF es eficaz desde el día 21 hasta el 56 PT y, además, presentó el mejor periodo residual, por lo cual constituye una excelente asociación para el control de garrapatas en animales en periodo de seca o aquellos en los que se respete el tiempo de retiro.

La reinfestación del medio ambiente juega un papel importante para la persistencia de $R$. microplus en los pastizales. Estos ectoparásitos oviponen más de 4000 huevos, pudiendo llegar a un 100\% de eclosión (Nuñez et al., 1982; Farias et al., 2007; Santos y Vogel, 2012); de allí la necesidad de evaluar el uso de ixodicidas que no solo controlen la presencia de garrapatas sobre el animal (evaluación en campo), sino, también, evaluar la eficiencia reproductiva y su eficacia sobre aquellas teleoginas expuestas al tratamiento (evaluación in vitro). Asimismo, es importante considerar que la resistencia a los ectoparasiticidas es un fenómeno presente en muchos países (FAO, 2003), por lo que se debe tener en cuenta la alternancia de drogas, el tiempo de residualidad y la correcta dosificación.

\section{ConClusiones}

- La formulación Fipronil 1\% + Abamectina $0.5 \%$ + Aceite de Neem $2 \%$ fue eficaz en la evaluación de cam- po (99.4 a $100 \%$ del día 7 al 28 de la aplicación) y $100 \%$ in vitro a las 24,48 y 72 horas de la colecta.

- La asociación Flumetrina 1\% + Eprinomectina $0.5 \%$ mostró una eficacia corta en campo (95.8 a $98.4 \%$ del día 7 al 14 de la aplicación) y a nivel in vitro fue eficaz (98-99\%) a las 48 y 72 horas de la colecta.

- La asociación Flumetrina 1\% + Fluazurón $2.5 \%$ mostró una eficacia del 94.4\% al día 14 del tratamiento; siendo su eficacia satisfactoria ( $\geq 95 \%)$ en campo a partir del día 21 hasta 56 días de la aplicación. Además, mostró el mayor periodo residual (97.3\%) hasta el día 56, y a nivel in vitro mostró una eficacia del $100 \%$ en las tres fechas poscolecta.

\section{Literatura Citada}

1. Aguirre DH, Gaido AB, Cafrune MM, Castelli ME, Mangold AJ, Guglielmone AA. 2005. Eprinomectin pour-on for control of Boophilus microplus (Canestrini) ticks (Acari: Ixodidae) on cattle. Vet Parasitol 127: 157-163. doi: 10.1016/j.vetpar.2004. 09.027

2. Baoliang P, Yuwan W, Zhende P, Lifschitz AL, Ming W. 2006. Pharmacokinetics of eprinomectin in plasma and milk following subcutaneous administration to lactating dairy cattle. Vet Res Commun 30: 263-270. doi: 10.1007/s11259-006-3230-7

3. Barriga O. 2002. Las enfermedades parasitarias de los animales domésticos en América Latina. Santiago de Chile: Germinal. 247 p.

4. Bezerra DS, Rodrigues B, Sanavria, Guimarães S. 2009. Avaliação da utilização de Nim (Azadirachta indica) para o controle parasitário em bovinos de produção leiteira em sistema orgânico. Rio de Janeiro: Boletim de Pesquisa e Desenvolvimento N. ${ }^{\circ} 47.36 \mathrm{p}$. 
5. Broglio-Micheletti SMF, NevesValente EC, De Souza AL, Da SilvaDias N; Girón-Pérez K, PrédesTrindade RC. 2009. Control de Rhipicephalus (Boophilus) microplus (Acari: Ixodidae) con extractos vegetales. Rev Colom Entomolo 35: 145-149.

6. Casas E, Trigueros A, Chávez A, Tang J, Ruiz F. 2009. Tratamiento y control de garrapata Boophilus microplus, a través de la combinación de fluazurón/ fipronil pour on, en bovinos de trópico, Pucallpa, Perú. [Internet]. Disponible en: http://www. agrovetmarket.com/pdf/ antiparasitario/Duotak\%20FF/ Duotak\%20FF\%20UNMSM.pdf

7. Castro-Janer E, Martins J, Mendes $M$, Namindome A, Klafke $G$, Schummaker T. 2010. Diagnoses of fipronil resistance in Brazilian cattle ticks (Rhipicephalus (Boophilus) microplus) using in vitro larval bioassays. Vet Parasitol 173: 300-306. doi: 10.1016/ j.vetpar.2010.06.036

8. Cid YP, Magalhães VS, Santos AL, Florêncio CN, Correia TR, Scott FB. 2012. Atividade in vitro do fipronil frente a diferentes populações de Rhiphicephalus Boophilus microplus (Acari: Ixodidae). Rev Bras Med Vet 34 (Supl 1): 5-10.

9. Cid YP, Magalhães VS, Silva DD, Lambert MM, Scott FB. 2010. Eficácia in vitro de lactonas macrocíclicas sobre teleóginas de Rhipicephalus (Boophilus) microplus (Acari: Ixodidae). Rev Bras Med Vet 32 (Suppl 1): 7-10.

10. Cordero del Campillo M, Rojo FD, Martínez AR, Sánchez MC, Hernández S, Navarrete I, Diez P, et al. 1999. Parasitología veterinaria. Madrid: McGraw Hill. 968 p.

11. Costa FB, Vasconcelos PSS, Silva AMM, Brandão VM, Da Silva IA, Teixeira WC, et al. 2008. Eficácia de fitoterápicos em fêmeas ingurgitadas de Boophilus microplus, provenientes da mesorregião oeste do Maranhão, Brasil. Rev Bras Parasitol Vet 17 (Supl 1): 83-86.
12. Cuore U, Cardozo H, Trelles A, Nari A, Solari MA. 2008. Características de los garrapaticidas utilizados en Uruguay. Eficacia y poder residual. Veterinaria 43: 13-24.

13. Cuore U, Solari MA, Cicero L, Trelles A, Gayo V, Nari A. 2009. Evaluación de los garrapaticidas actualmente disponibles en Uruguay para su utilización en los despachos de tropa. Veterinaria 45 : 23-30.

14. Da Silva HC. 2008. Parâmetros farmacocinéticos e atividade endectocida de uma nova formulação contendo avermectinas via tópica (pour-on), em bovines. Tesis Doctoral. São Paulo: Universidade Estadual Paulista. 111 p.

15. Davey RB, Ahrens EH, George JE, Hunter III JS, Jeannin P. 1998. Therapeutic and persistent e efficacy of fipronil against Boophilus microplus (Acari: Ixodidae) on cattle. Vet Parasitol 74: 261-276. doi: 10.1016/S03044017(97)00152-0

16. Díaz R. 2012. Mecanismos moleculares y bioquímicos de resistencia a acaricidas en la garrapata común de los bovinos Rhipicephalus microplus. Rev Colomb Cienc Anim 5: 72-81.

17. Drummond R, Ernst S, Trevino J, Gladney W, Graham O. 1973. Boophilus annulatus and Boophilus microplus: Laboratory test of insecticides. J Econ Entomol 66: 130-133.

18. El-Azazy OME, Lucas SF. 1996. The sterilizing effect of pour-on flumethrin on the camel tick, Hyalomma dromedarii (Acari: Ixodidae). Vet Parasitol 61: 339343. doi: 10.1016/0304-4017(95)00842-X

19. Elias VR. 1987. Avaliação do efeito carrapaticida de alguns piretróides sintéticos sobre o carrapato Amblyomma cajennense (Fabricius, 1787) (Acarina: Ixodidae). Tesis de Magíster. Rio de Janeiro: Universidade Federal Rural do Rio de Janeiro. $124 \mathrm{p}$.

20. Elliot M, Janes NF, Potter C. 1978. The future of pyrethroids in insect control. Annual Rev Entomol 23: 443-469. doi:10.1146/annurev.en.23.010178. 002303 
21. [EMEA] European Medicines Agency. 2005. Committee for medicinal products for veterinary use: Fluazuron. [Internet]. Disponible en: http://www.ema. europa. eu/docs/en_GB/document_library/ Maximum_Residue_Limits_-_Report/ 2009/11/WC500014283.pdf

22. [FAO] Food and Agriculture Organization of the United Nations. 1984. Tick and tick-borne disease control: a practical field manual. Rome: FAO. $621 \mathrm{p}$.

23. [FAO] Food and Agriculture Organization of the United Nations. 2003. Resistencia a los antiparasitarios. Estado actual con énfasis en América Latina. Roma: FAO. 51 p.

24. Farias MPO, Sousa DP, Arruda AC, Arruda MSP, Wanderley AG, Alves LC, Faustino MAG. 2007. Eficácia in vitro do óleo da Carapa guianensis Aubl. (andiroba) no controle de Boophilus microplus (Acari: Ixodidae). Rev Bras Plantas Med 9: 68-71.

25. Holdsworth PA, Kemp D, Green P, Peter RJ, De Bruin C, Jonsson N, Letonja T, et al. 2006. World Association for the Advancement of Veterinary Parasitology (W.A.A.V.P.) guidelines for evaluating the efficacy of acaricides against ticks (Ixodidae) on ruminants. Vet Parasitol 136: 29-43. doi: 10.1016/j.vetpar.2005.11.011

26. Lopes WD, Cruz BC, Teixeira WF, Felippelli G, Maciel WG, Buzzulini C, Gomes LV, et al. 2014. Efficacy of fipronil $(1.0 \mathrm{mg} / \mathrm{kg})$ against Rhipicephalus (Boophilus) microplus strains resistant to ivermectin $(0.63 \mathrm{mg} / \mathrm{kg})$. Prev Vet Med 115: 88-93. doi: 10.1016/ j.prevetmed.2014.04.009

27. Mansingh A, Williams LAD. 1998. Pesticidal potential of tropical plants. II. Acaricidal activity of crude extracts of several Jamaican plants. Insect Sci Appl 18: 658-664. doi: 10.1017/S1742758400007797

28. Manual Merck de Veterinaria. 2000. $5^{\mathrm{a}}$ ed. Barcelona: Océano. 758 p.
29. Martínez SS, Van Endem HF. 1999. Sublethal concentrations of azadirachtin affect food intake, conversion efficiency and feeding behaviour of Spodoptera littoralis (Lepidoptera: Noctuidae). Bull Entomol Res 89: 65-71.

30. Martínez SS. 2002. O NIM - Azadirachta indica: natureza, usos múltiplos, produção. Londrina, Brasil: Instituto agronômico do Paraná (IAPAR). 142 p.

31. Mekonnen S. 2000. Efficacy of flumethrin $1 \%$ pour-on against ticks on cattle under field conditions in Ethiopia. Onderstepoort J Vet Res 67: 235-237.

32. Municipalidad Provincial de Oxapampa. 2010. Plan de desarrollo concertado del distrito de Oxapampa 2009-2021. [Internet]. Disponible en: http://www.munoxapampa.com/ plandesarrollo/PlanDesarrolloConcertadoDistritoOxapampa.pdf

33. Muñoz CM. 2002. Antiparasitarios externos. En: Botana LM, Landoni F, Jiménez $\mathrm{G}$ (eds). Farmacología y terapéutica veterinaria. Madrid: McGrawHill Interamericana. p 505-516.

34. Nuñez J, Muñoz M, Moltedo H. 1982. Boophilus microplus: La garrapata común del ganado vacuno. Buenos Aires: Hemisferio Sur. 184 p.

35. Oliveira PR, Bechara GH, Marin MA, Camargo MI. 2009. Action of the chemical agent fipronil on the reproductive process of semi-engorged females of the tick Rhipicephalus sanguineus (Latreille, 1806) (Acari: Ixodidae). Ultrastructural evaluation of ovary cells. Food Chem Toxicol 47: 1255-1264. doi: 10.1016/j.fct.2009. 02.019

36. Oliveira PR, Calligaris IB, Roma GC, Bechara GH, Pizano MA, Camargo MI. 2012. Potential of the insect growth regulator, fluazuron, in the control of Rhipicephalus sanguineus nymphs (Latreille, 1806) (Acari: Ixodidae): determination of the LD95 and LD50. Exp Parasitol 131: 35-39. doi: 10.1016/ j.exppara.2012.02.023 
37. Penrroz H. 2009. Evaluación de fipronil (Frenil®) contra Rhipicephalus sanguineus (Latreille, 1806) en perros infestados en forma experimental. Tesis de Médico Veterinario. Chillán, Chile: Universidad de Concepción. 48 p.

38. Pereira JR. 2009. The efficiency of avermectins (abamectin, doramectin and ivermectin) in the control of Boophilus microplus, in artificially infested bovines kept in field conditions. Vet Parasitol 162: 116-119. doi: 10.1016/j.vetpar. 2009.02.014

39. Perez-Cogollo LC, Rodriguez-Vivas RI, Ramirez-Cruz GT, Miller RJ. 2010. First report of the cattle tick Rhipicephalus microplus resistant to ivermectin in Mexico. Vet Parasitol 168: 165-169. doi: 10.1016/j.vetpar.2009. 10.021

40. Plumb DC. 2010. Manual de farmacología veterinaria. $6^{a}$ ed. Buenos Aires: Inter-Médica. $1239 \mathrm{p}$.

41. Prullage J, Tran H, Timmons P, Harriman J, Chester S, Powell K. 2011. The combined mode of action of fipronil and amitraz on the motility of Rhipicephalus sanguineus. Vet Parasitol 179: 302-310. doi: 10.1016/ j.vetpar.2011.03.041

42. Rodríguez-Vivas RI, Arieta-Román RJ, Pérez-Cogollo LC, RosadoAguilar JA, Ramírez-Cruz GT, Basto-Estrella G. 2010. Uso de lactonas macrocíclicas para el control de la garrapata Rhipicephalus (Boophilus) microplus en el ganado bovino. Arch Med Vet 42: 115-123.

43. Rodríguez A, Rodríguez C, Cruz A. 2010. Efecto ixodicida de los extractos etanólicos de algunas plantas sobre garrapatas Rhipicephalus (Boophilus) microplus. Rev MVZ Córdoba 15:21752184.

44. Rojas M. 2004. Nosoparasitosis de los rumiantes domésticos peruanos. $2^{\mathrm{a}}$ ed. Lima: Maijosa. $146 \mathrm{p}$.

45. Santamaría M, Soberanes N, Fragoso H, Martins J, Cordovés C. 2003. Avaliação in vitro de uma cepa de cam- po de Boophilus microplus (Acari: Ixodidae) resistente à amitraz. Cienc Rural 33: 737-742. doi: 10.1590/S010384782003000400024

46. Santos FC, Vogel FS. 2012. Avaliação in vitro da ação do óleo essencial de capim limão (Cymbopogon citratus) sobre o carrapato bovino Rhipicephalus (Boophilus) microplus. Rev Bras Plantas Med 14: 712-716.

47. Shoop WL, Mrozik H, Fisher MH. 1995. Structure and activity of avermectins and milbemycins in animal health. Vet Parasitol 59: 139-156. doi: 10.1016/0304-4017(94)00743-V

48. Stendel W, Hamel H, Sieveking H, Brühne D. 1992. Analytical determination of distribution of flumethrin on the body surface of cattle following topical pour-on application. Vet Parasitol 42: 137-143. doi: 10.1016/0304-4017 (92)90109-M

49. Tang PJ, Ruiz HF, Rodríguez IL. 2008. Evaluación de eficacia y tolerancia de una solución topical externa sobre la base de flumetrina al $1 \%$ (Ectickol® Pour On) para el control de infestaciones por Microthoracius ovinus en alpacas de la Sierra Central. [Internet]. Disponible en: http:// www.agrovet market.com/pdf/ antiparasitario/Ectickol\%20Pour\%20On/ 2\%20Ectickol\%2 0Pour\% 20On\%20 Alpaca.pdf

50. Urquhart GM, Armour J, Duncan JL, Dunn AM, Jennings FW. 2001. Parasitología veterinaria. $2^{\mathrm{a}}$ ed. Zaragoza: Acribia. 355 p.

51. Villar D, Olivera M, Dídier R, Chaparro J. 2012. Aproximación al tema de residuos antimicrobianos y antiparasitarios en leche: Límites permisibles y tiempos de retiro. Medellín, Colombia: Biogénesis. $80 \mathrm{p}$.

52. Williams LAD, Mansingh A. 1996. The insecticidal and acaricidal actions of compounds from Azadirachta indica (A. Juss.) and their use in tropical pest management. Integr Pest Manage Rev 1: 133-145. 
53. Wood IB, Amaral NK, Barden K, Duncan JL, Kassai T, Malone JB, Pankavich JA, et al. 1995. World Association for the Advancement of Veterinary Parasitology (W.A.A.V.P.).
Second edition of guidelines for evaluating the efficacy of anthelmintics in ruminants (bovine, ovine, caprine). Vet Parasitol 58: 181-213. doi: 10.1016/03044017(95)00806-2 